\title{
2
}

\section{Unlocking the Design Potential of Rapid Manufacturing}

Richard Hague

Loughborough University

\subsection{Introduction}

One of the principal advantages of taking an additive (Rapid Manufacturing) approach to manufacturing over more conventional subtractive or formative methods comes not from manufacturing approach per se but from the dramatic advantages that are possible in the area of design. This potential for radically different design methodologies is one of the major drivers for the development of Rapid Manufacturing systems and materials and is a powerful reason why some organisations are able to put up with the sometimes severe limitations associated with current Rapid Prototyping (RP) systems to gain an advantage today.

The main benefit to be gained by taking an additive manufacturing approach (including most, but not all, of the currently available RP techniques) is the ability to manufacture parts of virtually any complexity of geometry entirely without the need for tooling. In conventional manufacturing, there is a direct link between the complexity of a part and its cost. In Rapid Manufacturing (RM), not only is complexity independent of cost but also the RM techniques are able to produce virtually any geometry. If this principal were extended to true manufacturing processes then the opportunities for product design and manufacturing are immense. 
This need for tooling in conventional manufacturing represents one of the most restrictive factors for today's product development. The absence of tooling within the additive manufacturing processes means that many of the restrictions of 'Design for Manufacture and Assembly' (DFMA) [1] that are essential in a modern manufacturing environment are no longer valid [2]. In injection moulding, for example, the need to consider the extraction of the part from the (usually expensive) tool takes an overriding precedence in the design of the part. Thus the high cost and need for tooling greatly limits product design and compromises have to be made. Without the need for tooling or necessity to consider any form of DFMA, the possibilities for design are literally only limited by imagination.

During the last few decades, designers have been educated to develop designs with restricted geometry so that parts can be made easily. The revolutionary aspect of Rapid Manufacturing will be that geometry will no longer be a limiting factor. Compounding the fact that as high volumes do not need to be manufactured to offset the cost of tooling then the possibilities for affordable, highly complex, custom parts become apparent. In theory, each part that is produced could be a custom part and thus there will be the potential to economically 'manufacture to a unit of one' [3]. The ability to produce whatever geometry that is created in a three-dimensional computer aided design (CAD) system actually means that one is entering a new dimension of 'Manufacture for Design' rather than the more conventional 'Design for Manufacture' philosophy [4].

This freedom of design is one of the most important features of RM and is extremely significant for producing parts of complex or customised geometries, which will result in reducing the lead-time and ultimately the overall manufacturing costs for such items. RM will affect manufacturers and customers alike. For manufacturers, costs will be dramatically reduced as no tooling is required and for customers, complex, individualised products will be cost-effectively made that can be configured to personal use, thus giving the potential for much greater customer satisfaction [5].

Rapid Manufacturing will enable fast, flexible and reconfigurable manufacturing to occur that will have enormous benefits to manufacturers and consumers. The elimination of tooling and the subsequent removal of many DFMA criteria will realise significant benefits in the design, manufacture and distribution of a part or components, including:

- Economic low-volume production

- Increased flexibility and productivity

- Design freedom

The subject of 'Design for Manufacture' is potentially broad. However, this chapter will concentrate on the 'freedom of design' aspects and will give 
details of specific areas of design that are only enabled by taking an additive approach to manufacturing.

\subsection{Potential of Rapid Manufacturing on Design}

The main feature of RM processes is the ability to produce parts of virtually any shape complexity without the need for any tooling. The impact of this factor on the validity of guidelines that designers comply with when they are designing for manufacture and assembly are discussed below.

\subsubsection{Conventional 'Design for Manufacture' (DFM)}

DFM is a philosophy or mind-set in which manufacturing input is used at the earliest stages of design in order to design parts and products that can be produced more easily and more economically. DFM is any aspect of the design process in which the issues involved in manufacturing the designed object are considered explicitly with a view to influencing the design. Some principals are used for efficient manufacturing, such as: developing a modular design, using standard components and designing for multi-use and to be multi-functional. By far the most important principle is to design for ease of manufacture and fabrication, which could be different depending on the manufacturing processes adopted. These guidelines are well documented elsewhere $[1,6]$.

For years, designers have been restricted in what they can produce as they have generally had to design for manufacture - i.e. adjust their design intent to enable the component (or assembly) to be manufactured using a particular process or processes. In addition, if a mould is used to produce an item, there are therefore automatically inherent restrictions to the design imposed at the very beginning.

As the range of plastic products being produced by RP and RM processes are quite comparable with those of injection moulding of plastics, some of the rules necessary for injection moulding are given here in order to provide a basis for the consideration of design rules for Rapid Manufacturing. These include:

1. Draft angles. These are important for ease of removal of parts from moulds. The inclusion of draft angles at the design stage is very important, but often omitted.

2. Minimising re-entrant features. 'An easy to manufacture part' must be easily ejected from the mould. Designing undercuts requires the use of side cores. This in turn will require moving parts in the dies that add to the tooling costs considerably. Some parts containing features such as blind 
holes and galleries are impossible to manufacture without using very complex and expensive tooling arrangements.

3. Wall thickness consideration. Components with thin walls solidify faster, hence reducing warpage and production costs.

4. Uniform wall thickness. Non-uniform wall thickness will result in compression and expansion of molecules, resulting in compressive and tensile stresses. The stress in turn will result in cracks, crazing or fractures of moulded parts.

5. Minimising weld lines. When different flow fronts (due to obstruction within the mould or various gates) meet each other, this creates weld or fusion lines. These are a source of weakness within the part and should be minimised during design.

6. Avoiding sharp corners. These will provide tensile, compressive and shear stress on the moulded parts, which in turn will become stress concentration points, leading to part failure.

7. Ejection pin marks and gate marks. These could have an adverse aesthetic effect on the injection-moulded part. However, with adequate consideration their impact could be minimised.

8. Parting line. The direction of mould closure and parting line is also crucial in tooling and injected parts. Much consideration and deliberation is needed for their selection.

9. Minimising sink marks. These are formed when a thin section becomes solid sooner than a developed thicker section. Sink marks could be less apparent by adequate consideration during design.

\subsubsection{Conventional Design for Assembly (DFA)}

By adopting DFA guidelines at the design stage, significant reductions in manufacturing cost and improvements in the ease of assembly can be achieved [7]. A few of these guidelines are briefly given here [1,6]:

1. Reducing parts count. Eliminating unnecessary parts, combining parts or eliminating or reducing the number of fasteners could achieve this.

2. Reducing handling time. A few simple, logical and effective rules, such as avoiding tangling and nesting parts or using easy-to-handle symmetrical parts, would result in a more efficient assembly.

3. Ease of insertion. This involves designing parts that are easy to align, easy to insert and self-locating with no need to be held in place before insertion of the next part.

\subsubsection{Impact of RM on DFM and DFA}

As the first RM processes will most probably be plastic processing systems, the most immediate competition will be with injection moulding. RM, unlike 
injection moulding, is a tool-less process, which does not involve any melting and subsequent solidification of materials within the confines of a tool. Therefore, considerations for constant wall thickness (to aid the flow of material), avoidance of sharp corners and minimising weld lines, sink marks, ejection pins, gates marks and draft angles will no longer need to be considered.

However, the significant impact of RM will be on the guidelines associated with minimising complex geometries and features such as undercuts, blind holes, screws, etc. Incorporating such features in conventional injection moulding is not impossible but often requires expensive tooling, extensive tool set-ups, testing runs and prototyping. This inevitably leads to undesirable lead-times and costs. Also, any simple modification in design requires a new set of tooling. However, as RM is a tool-less process, the part complexity is not important and any complex shapes or features produced by CAD can be directly translated into the final product. This is in marked contrast to conventional manufacturing processes.

Also, in injection moulding, the selection of the correct location for the split line - in particular for asymmetrical and complex-shaped components is quite difficult and is largely dependent on the experience of the tool designer. However, by adopting RM processes and not using any tooling, designers will be entirely freed from this task.

By using RM technologies, it will be possible to reduce the number of parts within an assembly. Therefore, the most important DFA guideline, which concerns the reduction in part count, is easily achievable. In theory it is possible to reduce the number of parts to just one, though in practice this may not feasible as parts are generally not being used in isolation and their interaction with other components would impose limitations on a part's count.

Thus, with the advent of the Rapid Manufacturing techniques, there is the potential for many of the current obstacles to be removed. The following sections discuss the design freedoms afforded by RM and also deal with some potential problems that are likely to occur with the onset of Rapid Manufacturing in general.

\subsection{Geometrical Freedom}

As discussed, one of the major benefits of some additive manufacturing processes is that it is possible to make virtually any complexity of geometry at no extra cost. This is virtually unheard of, as in every conventional manufacturing technique there is a direct link from the cost of a component to the complexity of its design. Therefore, for a given volume of component, it is effectively possible to get the geometry (or complexity) for 'free', as the 
costs incurred for any given additive manufacturing technique are usually determined by the time to build a certain volume of part, which in turn is determined by the orientation that the component is built in.

Areas of particular interest that are enabled by the freedoms afforded by RM include:

- Design complexity/optimisation

- Parts consolidation

- Body-fitting customisation

- Multiple assemblies manufactured as one

These areas are discussed in greater detail in the following sections.

\subsection{Design Complexity/Optimisation}

The design freedoms afforded by RM will enable increasingly complex designs to be realised that are fully optimised for the function that they are required for. Design optimisation is common in the construction industry where optimal structures for bridges and buildings are derived using optimisation techniques and then subsequently fabricated. For example, Figure 2.1 shows the proposed Beijing National Stadium, which has been designed by Arup for the 2008 Olympics. This building has been designed with a combination of design optimisation and genetic algorithms to produce a truly unique structure, but one that is structurally sound.

It is proposed that, due to the freedoms of design afforded by RM, this approach can be used much more extensively for product design - this approach is less common in the product design arena as the optimised design will often prove impossible to make due to DFM criteria. This is one of the main stumbling blocks for so-called Knowledge-Based Engineering (KBE) systems that often have finite element analysis (FEA) as the kernel.

Initial work at Loughborough University has investigated the use of design optimisation to create complex internal structures. Figure 2.2 illustrates a

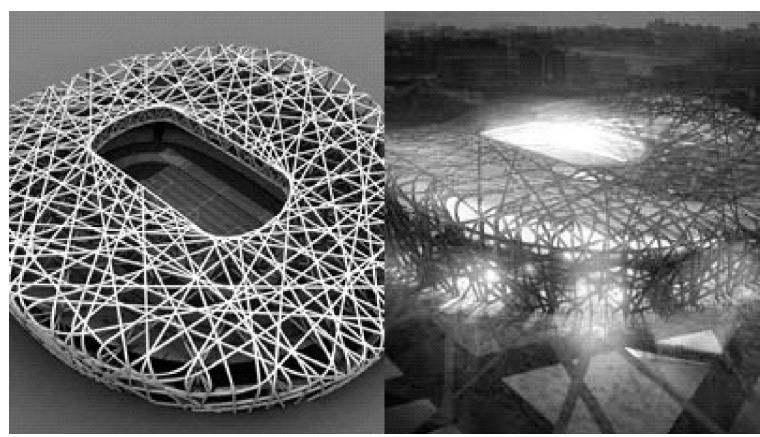

Figure 2.1 Proposed Beijing National Stadium designed by Arup (8) 


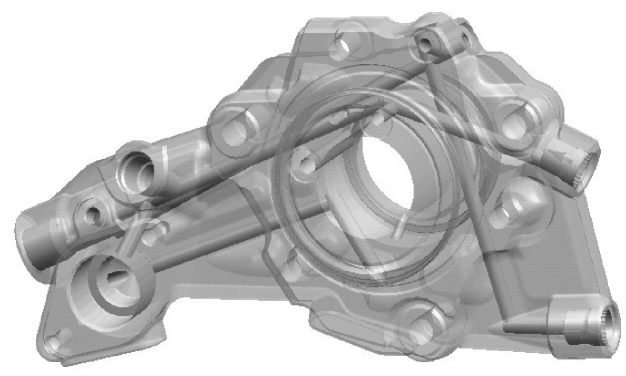

Figure 2.2 Conventional front plate
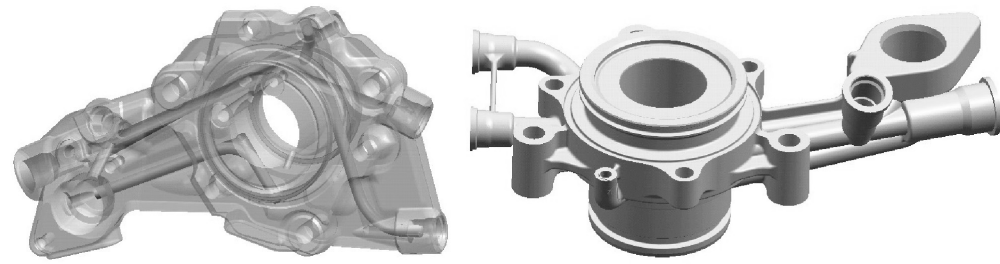

Figure 2.3 Optimised flow channels and design optimised front plate

diesel front plate manufactured by Delphi Diesel Systems showing internal flow channels that have to be conventionally gun-drilled.

A consideration has been made as to what the design would be like if it were possible to manufacture this by RM. Figure 2.3 shows firstly the flow channels that would be manufacturable by RM followed by a design that is optimised for minimal weight that has been constructed around the flow lines. This approach represents a new philosophy to product design that will have radical implications for the performance of items in the future - only RM allows this.

\subsubsection{Part Consolidation}

One of the most important opportunities to arise from the ability to 'manufacture for design' comes from the very real potential to consolidate many components into one. This reduction of assemblies has tremendous implications, not just for the actual assembly of the components and the consequent cost savings that can be gained but also from the potential to maximise a design of a product for the purpose in mind and not to have to compromise the design for manufacturing and assembly reasons. Figure 2.4 shows an example of part consolidation where a complex ducting channel assembly has been consolidated into just one part. Such an approach is currently being utilised by US aircraft manufacturers for the production of ducting (on SLS, or selective laser sintering, machines) for use in fighter aircraft. 

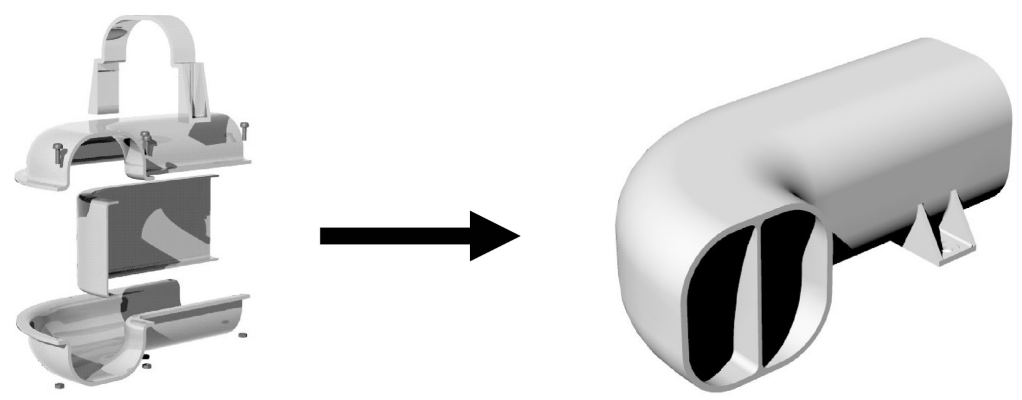

Figure 2.4 Example of parts consolidation in aircraft ducting (9)
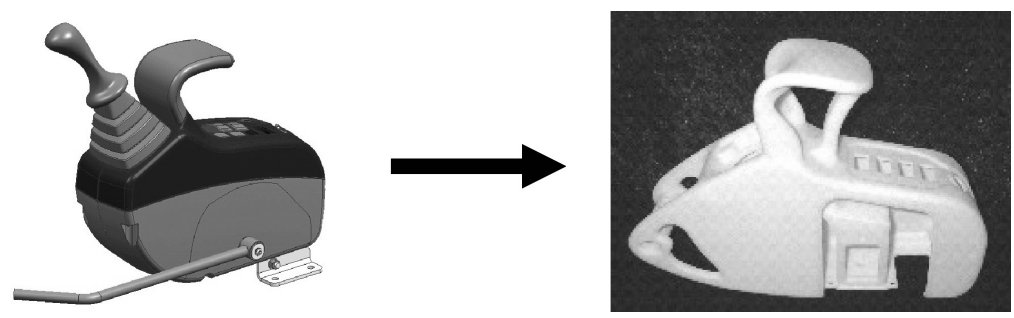

Figure 2.5 Consolidation of the control pod

Work has been undertaken at Loughborough into the concept of parts consolidation. Figure 2.5 shows how an assembly of over 25 parts has been consolidated into just one piece (with an extra cover) and then manufactured by stereolithography (SL). Early calculations suggest that, not withstanding the material properties, the SL pod would be commercially viable due to the low number that would be manufactured and also the advantages that are gained by consolidating the assembly into just one part.

Another example of parts consolidation from Loughborough University involves the redesign and manufacture of car door handles with Jaguar Cars. An initial assembly comprising eleven components made from eight different materials was simplified to a single component made from a single material that was manufactured by SLS. In this case the work showed how the design freedoms afforded by RM could ease the end-of-life recycling.

\subsubsection{Body Fitting Customisation}

The production of body fit parts and core customisation is not novel. However, the creation of customised products using conventional skills and technologies, especially truly body fitting customised products, has traditionally been very labour-intensive and essentially craft-based. Thus, partly due to the costs of labour, customised products are usually out of reach of the general public, who are forced to buy mass-produced goods. 
As true customisation is at present not feasible for the mass market, the concept of 'mass customisation' is currently employed to give some degree of customisation. However, mass customisation is really achieved by 'modularisation' - the production of modules that can be bolted together in varying configurations, which that gives the economies of mass production but allows some choice in the product. By using management techniques such as postponement, the decision of how the final product is configured can be delayed to allow for greater degrees of customisation. However, standard manufacturing techniques are still employed (for the modules) and therefore there is still the need for costly tooling.

However, through the adoption of reverse engineering and Rapid Manufacturing, the era of cost-effective customisation for the masses is not far off. With the advent of RM the production method and processes involved for customised parts would not change from part to part. Thus, the economic argument for providing core (body fitting) customisation is greatly enhanced. This approach is already commercial reality as Siemens and Phonak are using laser sintering and stereolithography to manufacture bespoke hearing aids for end use (see Chapter 12).

At Loughborough University, this approach is being further considered by the MANRM project (under a DTI Foresight Vehicle Initiative) where a methodology has been established for the capture of the correct deformed geometry that is required for creating the body fitting customised parts. This project is particularly focused on the aerospace/automotive sectors. Figure 2.6 shows a body fitting seat that has been produced for one of the project partners, MG Rover. The parts are then manufactured automatically using RM technologies that require no expensive tooling. In the near future, this work will lead to affordable custom fitting products for the general public.

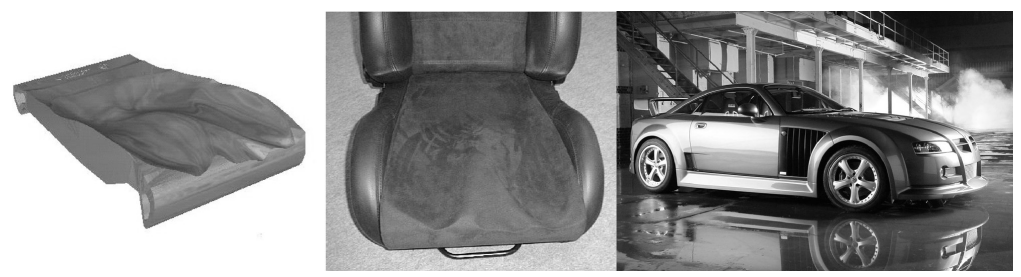

Figure 2.6 Body fitting seating platform and seat for the MG SV. (Reproduced with permission of MG Rover Group)

\subsubsection{Multiple Assemblies: Textiles}

An entirely novel area that has received virtually no consideration for RM, but one that has vast and exciting potential for future applications is that of smart textiles. Conventional sheet textiles, as with any other product, have to be constructed with the manufacturing process in mind and thus textiles are 


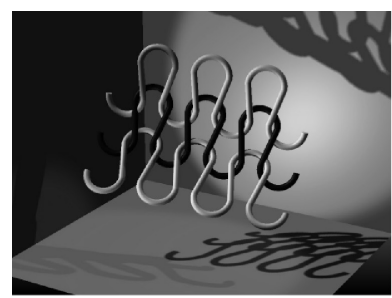

Conventional knitted weave

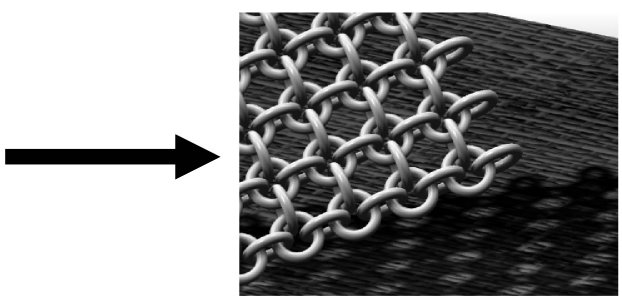

RM weave

Figure 2.7 Comparison of conventional versus RM textiles

fundamentally limited by the need to design for manufacture. Conventional fabric/textile construction uses centuries-old principles - the key for RM fabrics (at present) is to move from continuous fibres to individual links. This was first proposed by Jiri Evenhuis and Janne Kyttanen [10] in 1999. This is demonstrated in Figure 2.7.

However, there are many research issues that need to be overcome before RM produced garments are a reality. These research issues include:

- Link design

- Generation of three-dimensional data

- Lofting of data over conformal surfaces

- Very large data sets

- Collapsing of the structures for efficient manufacture

- Rapid Manufacturing process resolution

One of the most fundamental issues for creating body fitting RM textiles is the difficulty of using current CAD systems, which are not intended or capable of creating such complex assemblies. However, the Loughborough Rapid Manufacturing Research Group (in collaboration with Nottingham University Composite Materials Research Group) have developed a preliminary methodology to wrap textile links over complex surfaces and then manufacture them by SLS. This is shown in Figure 2.8.

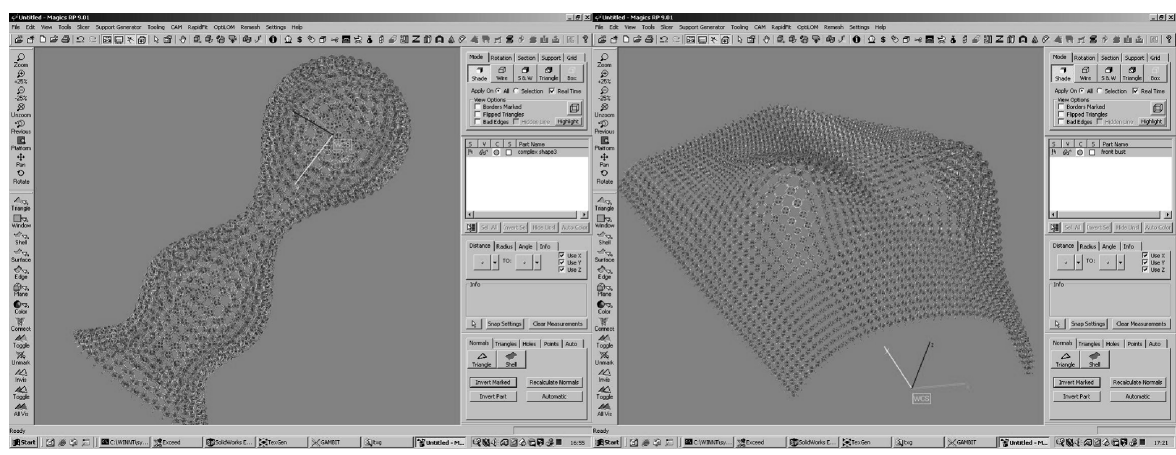

Figure 2.8 Wrapping of links over complex surfaces 


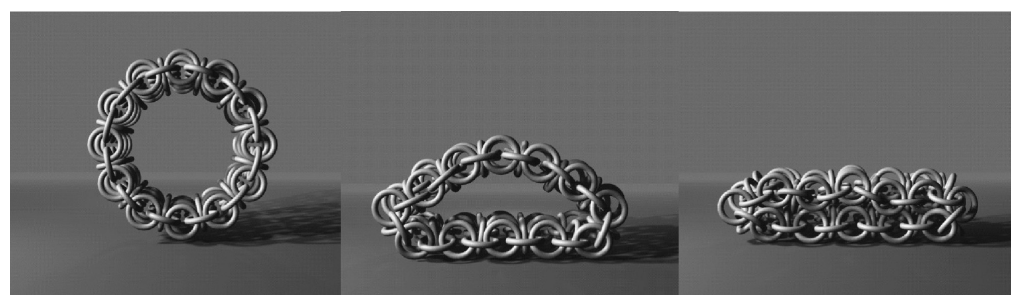

Figure 2.9 Structure collapsing for efficient manufacture

The current manufacture of all RM textile sheets, garments or products requires the design and CAD data to directly mimic the finished component in its three-dimensional form (as shown in Figure 2.8). Therefore, in order to fit the garment in the build envelope and also limit the $z$ height or number of layers (and thus minimise the cost), it is necessary to then collapse these data into a manufacturable form. Initial work has established a methodology for performing this for simple link constructions (as demonstrated in Figure 2.9), but much work is required to extend this to more complex constructions.

In summary, it is postulated that future RM systems will be capable of creating textiles directly and will be extended to the automated generation of the items that they are assembled into (e.g. clothing) using RM as the enabling technology. As a demonstration, Figure 2.10 shows an example of an RM dress that has been produced using current laser sintering technologies, which is the world's first fully conformal (body fitting) textile

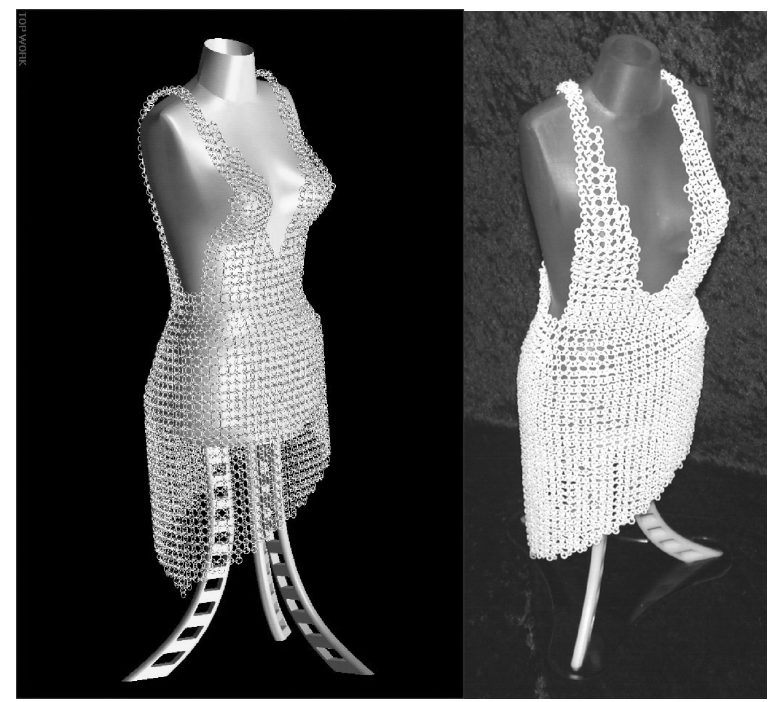

Figure 2.10 Examples of laser sintered fabrics produced at Loughborough 
garment to be produced directly in its assembled state. This, again, is only achievable by utilising an additive manufacturing approach.

Work is now underway aimed at investigating the potential for microlevel design and manufacture in the context of RM and initiating research into the automated knowledge-based generation of design optimised smart textiles producible via RM. In the future, there is much scope for:

- Seamless garments that can be manufactured fully assembled

- Variable 'weave'

- Products that transition from a solid to a textile configuration, thus giving possibilities for optimised footwear

- Smart textiles with built-in functionality (e.g. truly wearable computers, built-in chemical/biological detection, custom fitting armoured jackets, etc.)

\subsection{Material Combinations}

When objects are formed in moulds, they are generally formed in one homogeneous material. Even in the case of an over-moulded component, where there can be two or more homogeneous materials in one finished part, there is a definitive boundary between one material and the other. In the future, with some of the additive manufacturing processes there is the potential to mix and grade materials in any combination that is desired, thus enabling materials with certain properties to be deposited where they are needed [11,12].

The over-moulding technique is a classic example of how design can be influenced by the availability of a manufacturing technique. Overmoulding allows designers, within limits, the ability to produce parts that have added functionality and enhanced design. Indeed, the design of over-moulded components very often incorporates different material combinations to accentuate the design to the extent that designers are able to exploit the delineation of the different materials used to produce design features as well as extra functionality. This is perfectly illustrated by the simple case of a toothbrush - an everyday item that will often include over-moulding to give a handle that is stiff, with an over-moulded grip and a different material at the neck to give a flexible head. This is illustrated in Figure 2.11.

Given that RM potentially allows the development of multiple materials to be deposited in any location or combination that the designer requires, this has potentially enormous implications for the functionality and aesthetics that can be designed into parts. This concept of functionally graded materials (FGMs) is further discussed in Chapter 7. 


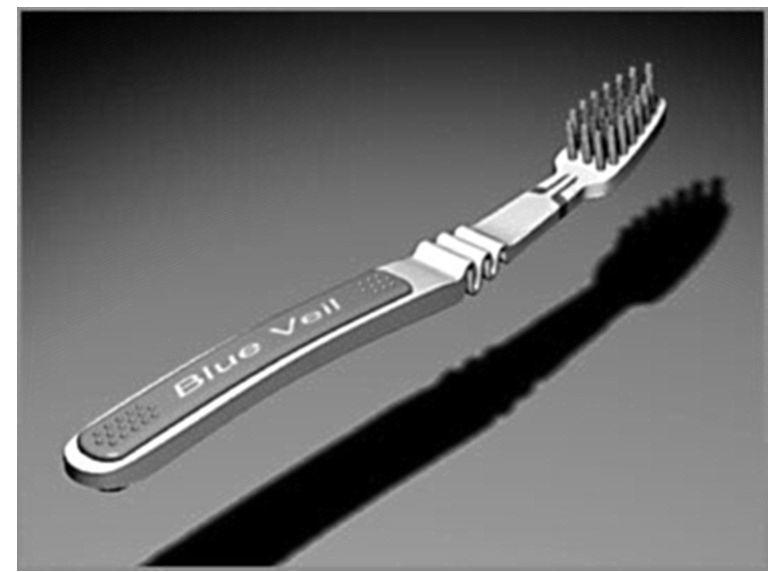

Figure 2.11 Example of how over-moulding improves design and functionality

\subsection{Summary}

The possibilities offered by Rapid Manufacturing are enormous. Suddenly, designers will be able to manufacture almost any shape that they come up with and will no longer be constrained by the necessity to produce parts in moulds. In addition, using processes such as the laser sintering of dissimilar powders, RM will provide designers with new and exotic materials not available to other manufacturing processes.

At the design phase, RM allows almost whatever shape is desired as the mould process will no longer limit design. This means objects can be designed with re-entrant features, no draft angles, unlimited wall thickness and increased complexity, with none of the limitations imposed by either the moulding process or the tool making process, as neither will be required.

One of the most profound implications of RM on design will be that, without the cost of tooling to amortise into the parts produced, each component can be different, potentially allowing for true mass customisation of each and every product. With developments in web-enabled software, high levels of computer literacy and Internet connectivity in the home, the technologies are not far from giving the consumer the ability to modify the design of the product they desire for themselves. Although some way off, it is conceivable that the consumer may - for a price - want to influence the design of their new sunglasses, mobile phone casing, steering wheel grip, surgical instrument, prosthetic part or favourite kitchen utensil, etc., and then send the data back to the manufacturer to be made for them.

RM will become more of a reality when the properties of the materials that are produced become more acceptable and consistent. This materials research is one of the main stumbling blocks to the adoption of these 
additive manufacturing techniques for end-use parts and is the subject of much current research. However, many organisations are willing to accept the materials limitations that are in evidence today to gain an advantage from the design possibilities.

\section{References}

1. Boothroyd, G., Dewhurst, P. and Knight, W. (1994) Product Design for Manufacture and Assembly, Marcel Dekker Inc., New York.

2. Mansour, S. and Hague, R. (2003) Impact of rapid manufacturing on design for manufacture for injection moulding, Proceedings of the Institution of Mechanical Engineers, Part B: Journal of Engineering Manufacture, 217(B4), 453-61.

3. UK manufacturing: we can make it better, Foresight Manufacturing 2020 Panel, Final Report, Findlay Publications, p. 12.

4. Campbell, R.I., Hague, R.J.M., Sener, B. and Wormald, P.W. (2003) The potential for the bespoke industrial designer, The Design Journal, 6(3), 24-34.

5. Hague, R.J., Campbell, R.I. and Dickens, P.M. (2003) Implications on design of rapid manufacturing, Proceedings of the Institution of Mechanical Engineers, Part C: Journal of Mechanical Engineering Science, 217(C1), 25-30.

6. Poli, C. (2001) Design for Manufacturing, A Structured Approach, Butterworth- Heinemann, Boston, Massachusetts.

7. Fox, S., Marsh, L. and Cockerham, G. (2001) Design for manufacture: a strategy for successful application to building, Construction Management and Economics, 19, 493-502.

8. www.arup.com

9. www.3dsystems.com

10. www.freedomofcreation.com

11. Anon (2001) The solid future of rapid prototyping, The Economist Technology Quarterly, 24 March 2001, pp. 47-9.

12. Jacobs, P.F. (2002) From stereolithography to LENS: a brief history of laser fabrication, International Conference on Metal Powder Deposition for Rapid Manufacturing, San Antonio, Texas, 8-10 April 2002. 